\title{
SSR profiling of potato cultivars resistant to pathogens
}

Gritsenko D.A. ${ }^{1,2 *}$, Aubakirova K.P. ${ }^{1}$, Pozharskiy A.S. ${ }^{1,2}$

${ }^{1}$ Institute of Plant biology and biotechnology, Almaty, Kazakhstan

${ }^{2}$ Al Farabi Kazakh National University, Almaty, Kazakhstan

*email: d.kopytina@gmail.com

Potato is among the most important vegetable crop in Kazakhstan. Genetic pool of potato consists of more than 2050 varieties, including $18 \%$ of Kazakhstani cultivars. In present study, SSR-genotyping of 42 Kazakhstani cultivars, 19 varieties of the world collection and 3 species of wild potatoes was carried out. 42 cultivars included resistant and highly resistant varieties to the late blight, early blight, potato wart and viral pathogens according to the results of field studies.

Genotyping was carried out using 24 SSR markers proposed in the new Potato genetic identity kit. The markers are evenly distributed over all potato chromosomes, every chromosome includes 2 markers with distance $10 \mathrm{cM}$ between each other. Fragment analysis was performed on Applied Biosystems 3500xL Genetic Analyzers.

Based on the results of phylogenetic analysis, a distinct cluster of Kazakhstani cultivars was determined. Polymorphic index content ranged from 0.465 to 0.875 . The least polymorphism was in the STG0025 marker; only 3 alleles were identified. The markers STG0001, STG0010, STM5114 had the highest polymorphism. As a result of the investigation, genetic profiles for 42 Kazakhstani cultivars were developed for markerassisted selection. 